Supporting Information to

\title{
How Big Is Big Enough? Effect of Length and Shape of Side Chains on the Single-Chain Enthalpic Elasticity of a Macromolecule
}

Zhonglong Luo ${ }^{1}$, Afang Zhang ${ }^{2}$, Yongming $\mathrm{Chen}^{3}$, Zhihao Shen ${ }^{4}$ and Shuxun Cui ${ }^{1 *}$

${ }^{1}$ Key Laboratory of Advanced Technologies of Materials, Ministry of Education, Southwest Jiaotong University, Chengdu 610031, China

${ }^{2}$ Department of Polymer Materials, College of Materials Science and Engineering, Shanghai University, Nanchen Road 333, Shanghai 200444, China

${ }^{3}$ School of Materials Science and Engineering, Sun Yat-sen University, Guangzhou 510275, China

${ }^{4}$ Beijing National Laboratory for Molecular Sciences, Key Laboratory of Polymer Chemistry and Physics of Ministry of Education, Center for Soft Matter Science and Engineering, College of Chemistry and Molecular Engineering, Peking University, Beijing 100871, China

*E-mail: cuishuxun@swjtu.edu.cn (S.C.). 
Table S1. The typical physical properties of polypropylene (PP) and polystyrene (PS). $T_{m}$ is the melting temperature, and $T_{g}$ is the glass transition temperature.

\begin{tabular}{|c|c|c|}
\hline Typical Value & PP & PS \\
\hline$T_{m} /{ }^{\circ} \mathrm{C}$ & 170 & 243 \\
\hline Tensile Strength $/ \mathrm{kg} \bullet \mathrm{cm}^{-2}$ & 300 & 590 \\
\hline$T_{g} /{ }^{\circ} \mathrm{C}$ & -10 & 100 \\
\hline
\end{tabular}

Flow of fluid

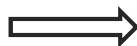

$$
F_{f}
$$

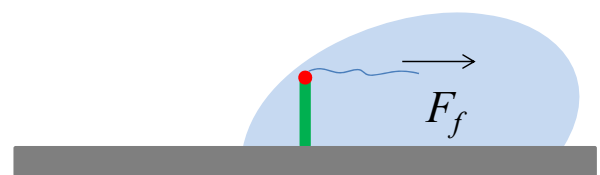

Substrate

Figure S1. A random-coiled polymer can be stretched into an extended conformation by a weak force via a fluid flow $\left(F_{f}\right)$.

Assuming that one end of the polymer was fixed, the random-coiled polymer will be stretched by the force exerted by the fluid flow. The force, $F_{f}$, can be calculated by the Stokes formula below:

$$
F_{f}=6 \pi \eta r v
$$


where $\eta$ is the viscosity coefficient of the fluid, $r$ is the radius of the cross-section of the polymer chain and $v$ is the velocity of the fluid. For the case of $r=0.2 \mathrm{~nm}$ and $v=$ $0.25 \mathrm{~m} / \mathrm{s}$, and water as the fluid at $20{ }^{\circ} \mathrm{C}\left(\eta=1.005 \times 10^{-3} \mathrm{~Pa} \cdot \mathrm{s}\right), F_{f}$ can be calculated to be $0.95 \mathrm{pN}$. When an organic solvent is used as the fluid, $F_{f}$ will be much smaller due to the much lower viscosity coefficient.
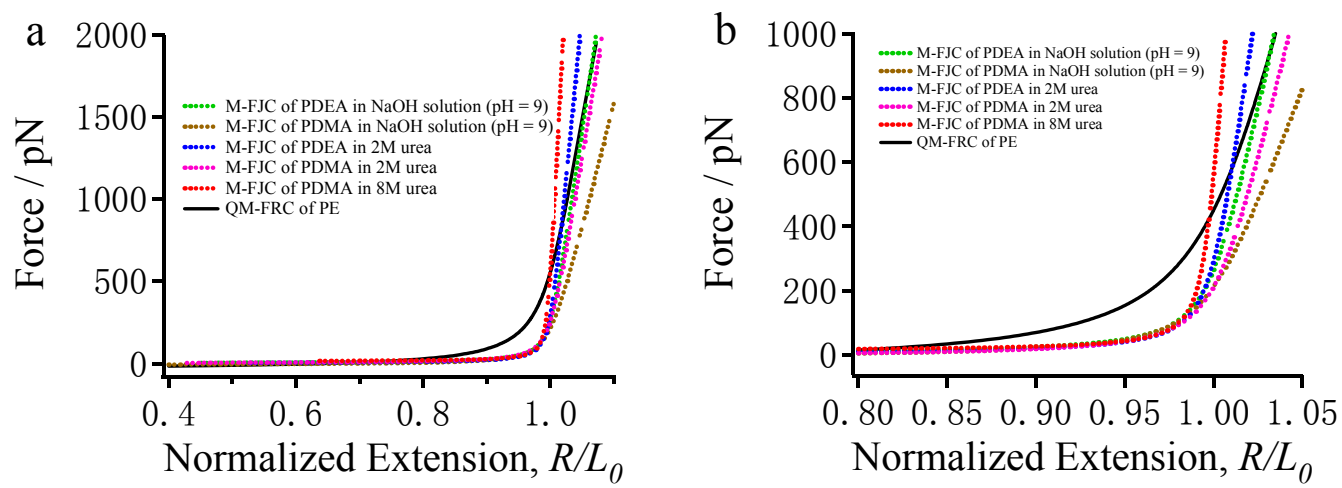

Figure S2. (a) Comparison of the fitting curves (QM-FRC fitting curve in this work and the M-FJC fitting curves from reference ${ }^{1}$ ). One can find that the polymer SCEE of PDEA and PDMA can be affected greatly by the aqueous solutions. (b) The fitting curves in (a) are magnified to show the details. 


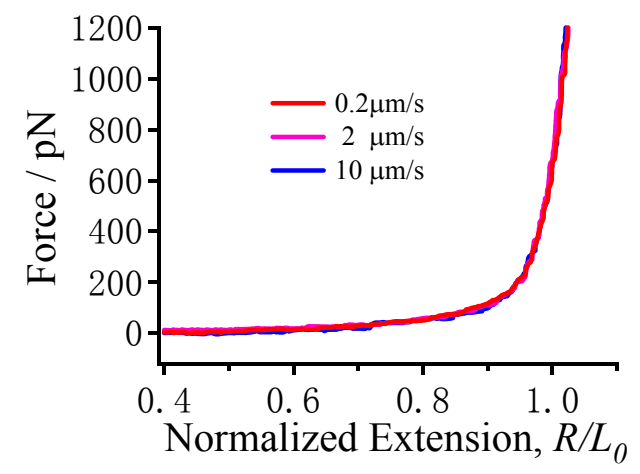

Figure S3. The normalized F-E curves of PG2 obtained in octane under different pulling speed.

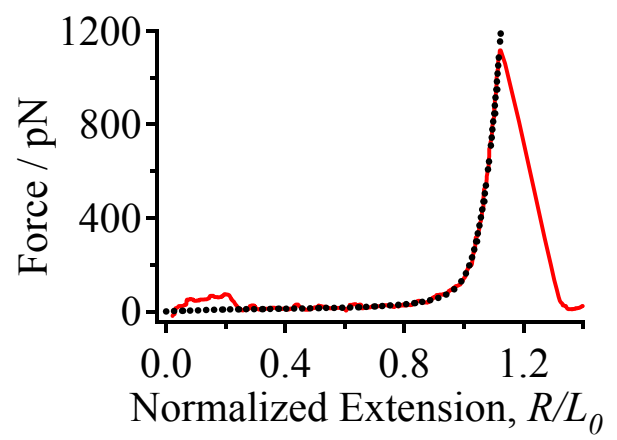

Figure S4. A typical F-E curve of PS obtained in octane (red line) with the QM-FRC fitting curve of $\mathrm{PE}\left(l_{b}=0.154 \mathrm{~nm}\right.$, dotted line). This result indicates that the QM-FRC model of PE can describe the SCEE of PS well. 

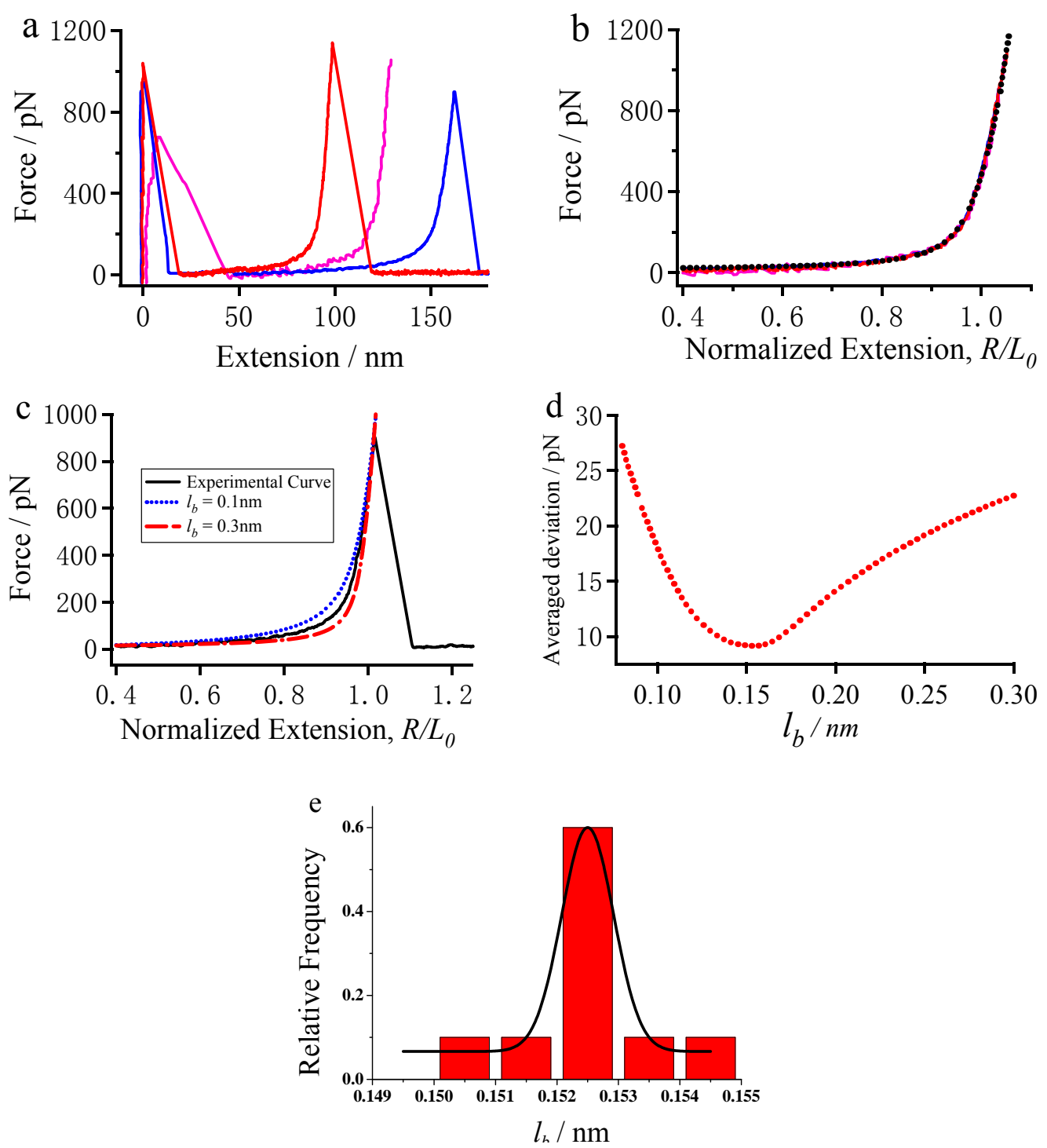

Figure S5. (a) The F-E curves of PVS obtained in nonane. (b) The normalized F-E curves of those shown in (a) and the QM-FRC fitting curve of PE $\left(l_{b}=0.1525 \mathrm{~nm}\right.$, dotted line). (c) Normalized F-E curves of PVS vs QM-FRC fitting curves with various $l_{b}$ values. (d) Averaged deviation of force between an experimental F-E curve of PVS showing in (c) and the QM-FRC fitting curve in the range of $R / L_{0}=0.6-1.04$, namely, $\left(\sum_{i=1}^{n}\left|F_{\text {exp }}-F_{f i t}\right|\right) / \mathrm{n}$, as a function of $l_{b}$. The saddle point is determined to be the optimum value of $l_{b}$ for the corresponding F-E curve in (c). (e) The histogram of $l_{b}$ of all F-E curves of PVS obtained in nonane. Gaussian fit shows that the most probable of $l_{b}$ is $0.1525 \mathrm{~nm}$ with a standard error of $2.2 \times 10^{-4} \mathrm{~nm}$. 

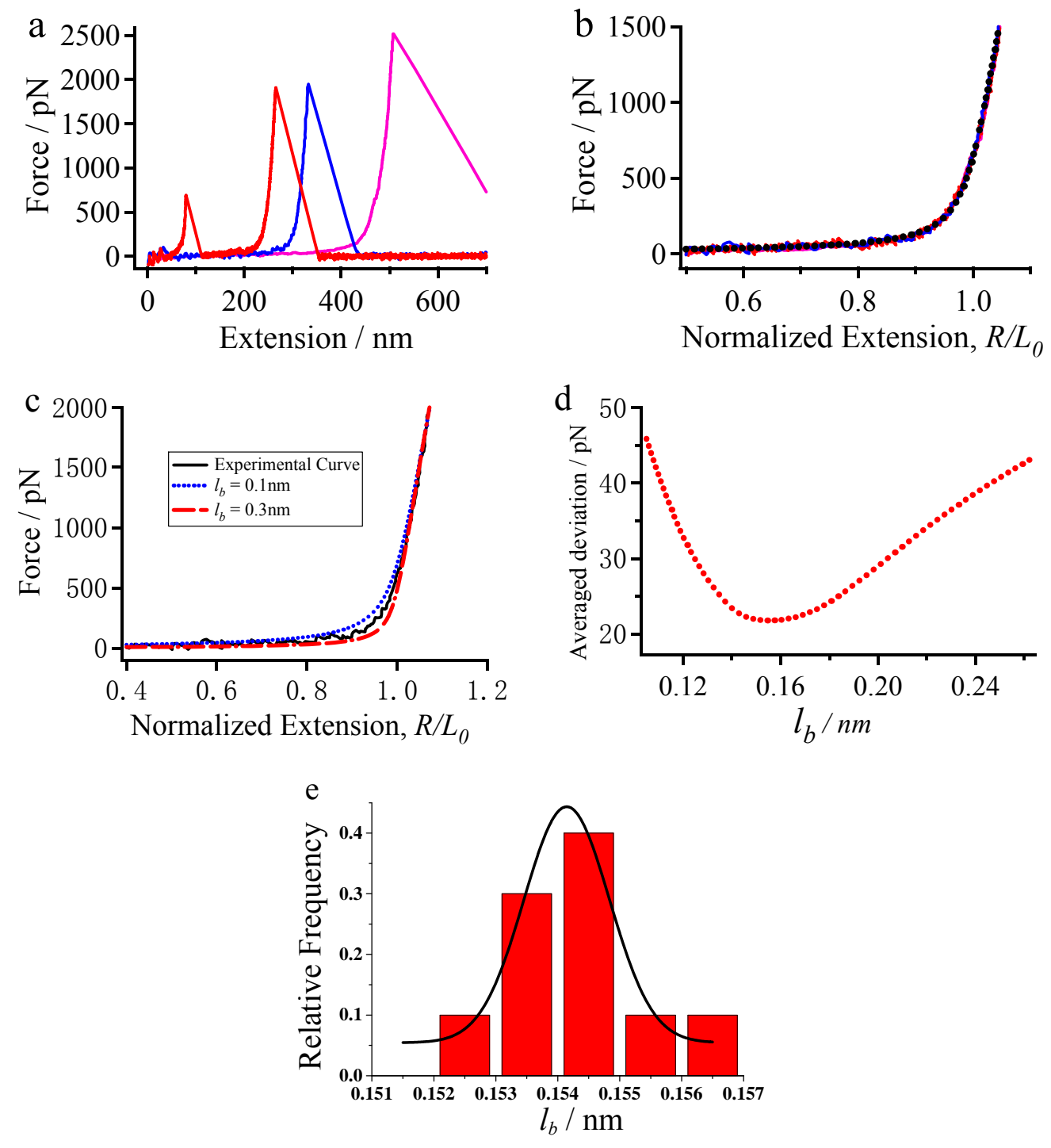

Figure S6. (a) The F-E curves of PGMA-g-PS 44 obtained in nonane. (b) The normalized F-E curves of those shown in (a) and the QM-FRC fitting curve of PE ( $l_{b}=0.154 \mathrm{~nm}$, dotted line). (c) Normalized single-molecule force curve of PGMA-g-PS 44 vs QM-FRC fitting curves with various $l_{b}$ values. (d) Averaged deviation of force between the experimental F-E curve of PGMA-g-PS 44 showing in (c) and the QM-FRC fitting curve in the range of $R / L_{0}=0.5-1.05$. The minimum deviation is obtained at $l_{b}=0.154 \mathrm{~nm}$. (e) The histogram of $l_{b}$ of all F-E curves of PGMA-g-PS 44 obtained in nonane. Gaussian fit shows that the most probable of $l_{b}$ is $0.1541 \mathrm{~nm}$ with a standard error of $1.2 \times 10^{-4} \mathrm{~nm}$. 

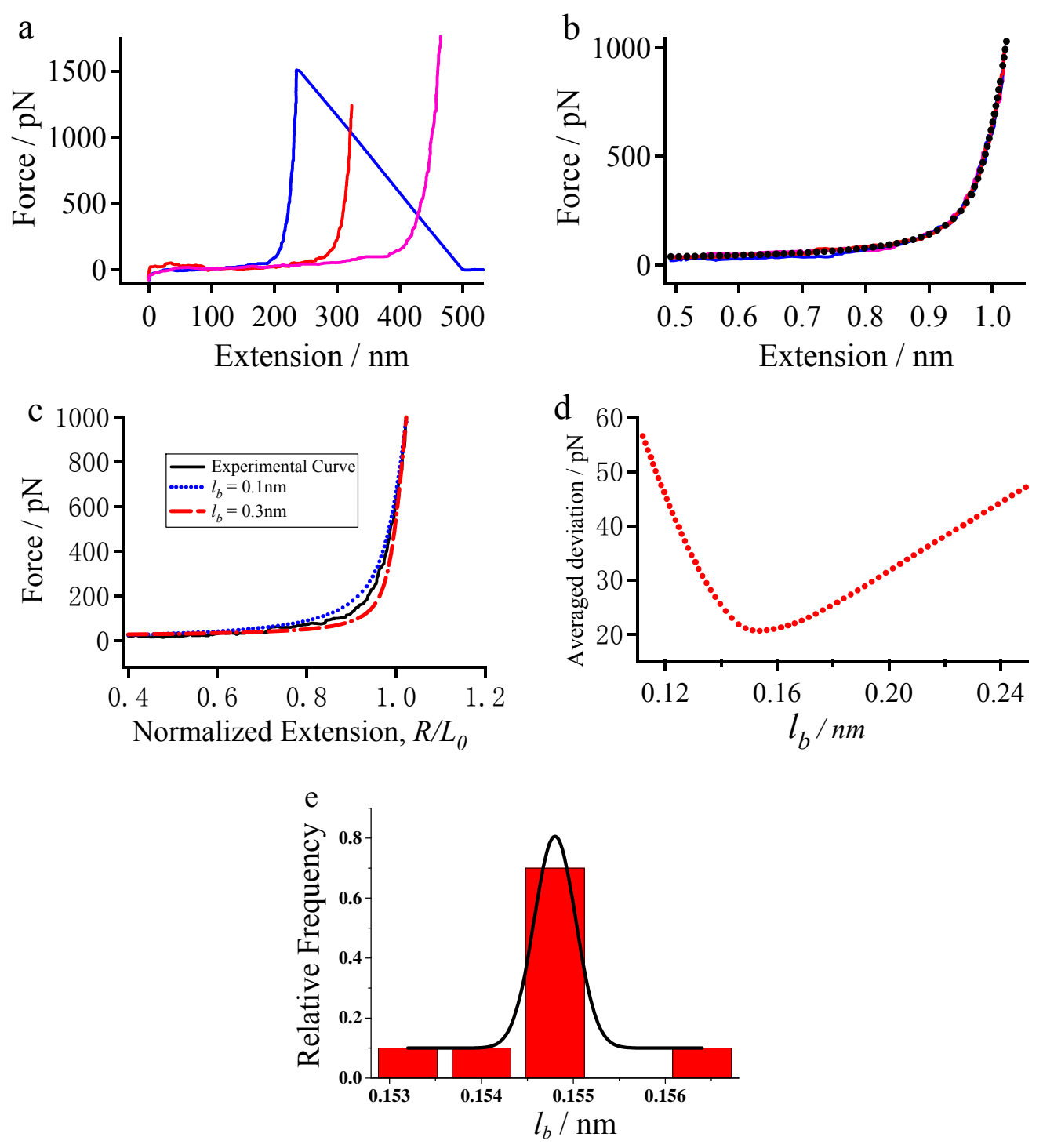

Figure S7. (a) The F-E curves of PGMA-g-PS 105 obtained in nonane. (b) The normalized F-E curves of those shown in (a) and the QM-FRC fitting curve of PE ( $l_{b}=0.155 \mathrm{~nm}$, dotted line). (c) Normalized single-molecule force curve of PGMA-g-PS 105 vs QM-FRC fitting curves with various $l_{b}$ values. (d) Averaged deviation of force between the experimental F-E curve of PGMA-g-PS 105 showing in (c) and the QM-FRC fitting curve in the range of $R / L_{0}=0.5-1.04$. The minimum deviation is obtained at $l_{b}=0.155 \mathrm{~nm}$. (e) The histogram of $l_{b}$ of all F-E curves of PGMA-g-PS 105 obtained in nonane. Gaussian fit shows that the most probable of $l_{b}$ is $0.1548 \mathrm{~nm}$ with a standard error of $4.9 \times 10^{-3} \mathrm{~nm}$. 

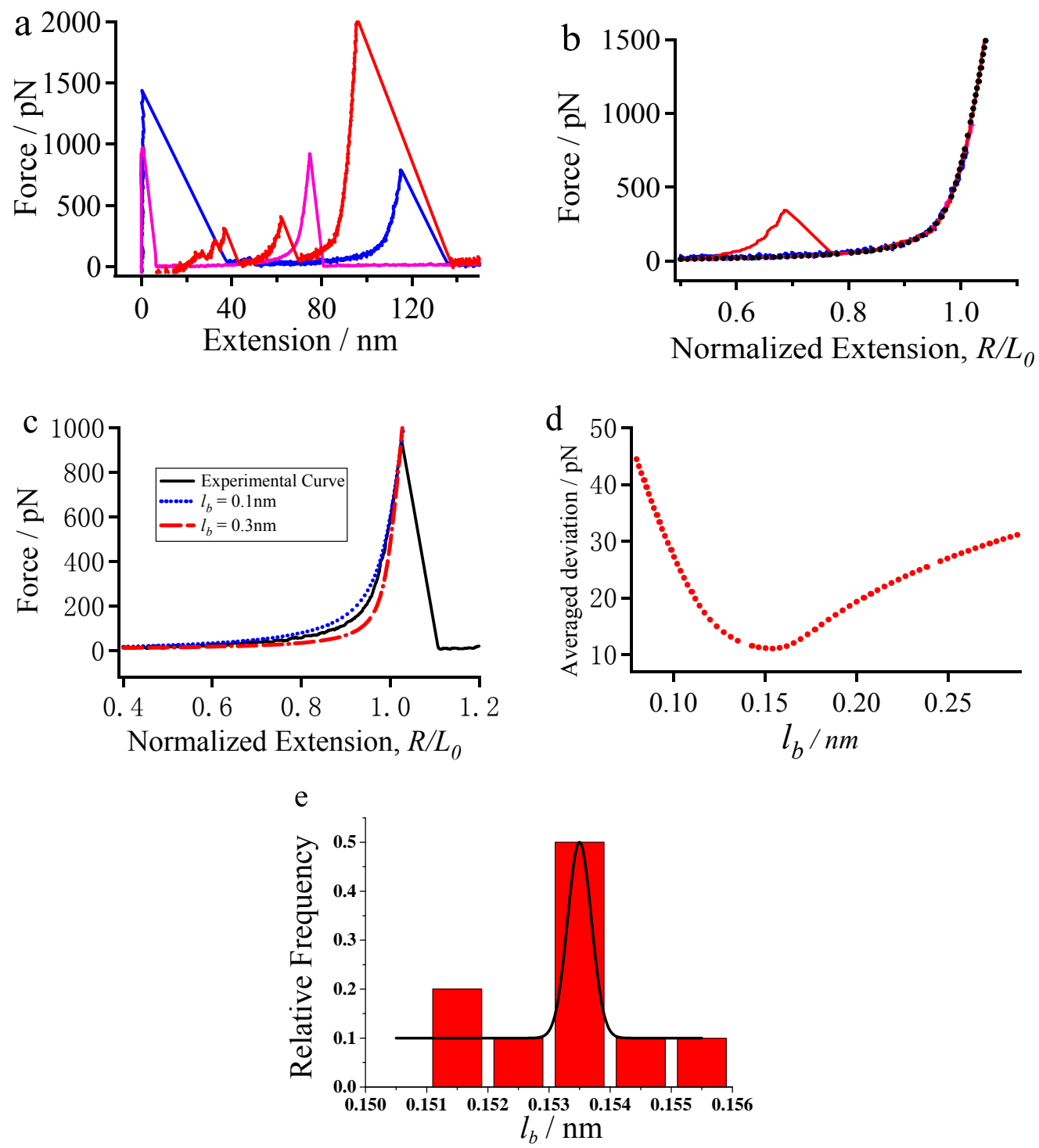

Figure S8. (a) The F-E curves of PBCPS obtained in nonane. (b) The normalized F-E curves of those shown in (a) and the QM-FRC fitting curve of PE $\left(l_{b}=0.153 \mathrm{~nm}\right.$, dotted line). (c) Normalized single-molecule force curve of PBCPS vs QM-FRC fitting curves with various $l_{b}$ values. (d) Averaged deviation of force between the experimental F-E curve of PBCPS showing in (c) and the QM-FRC fitting curve in the range of $R / L_{0}=0.5-1.05$. The minimum deviation is obtained at $l_{b}=0.153 \mathrm{~nm}$. (e) The histogram of $l_{b}$ of all F-E curves of PBCPS obtained in nonane. Gaussian fit shows that the most probable of $l_{b}$ is $0.1535 \mathrm{~nm}$ with a standard error of $3.5 \times 10^{-4} \mathrm{~nm}$. 

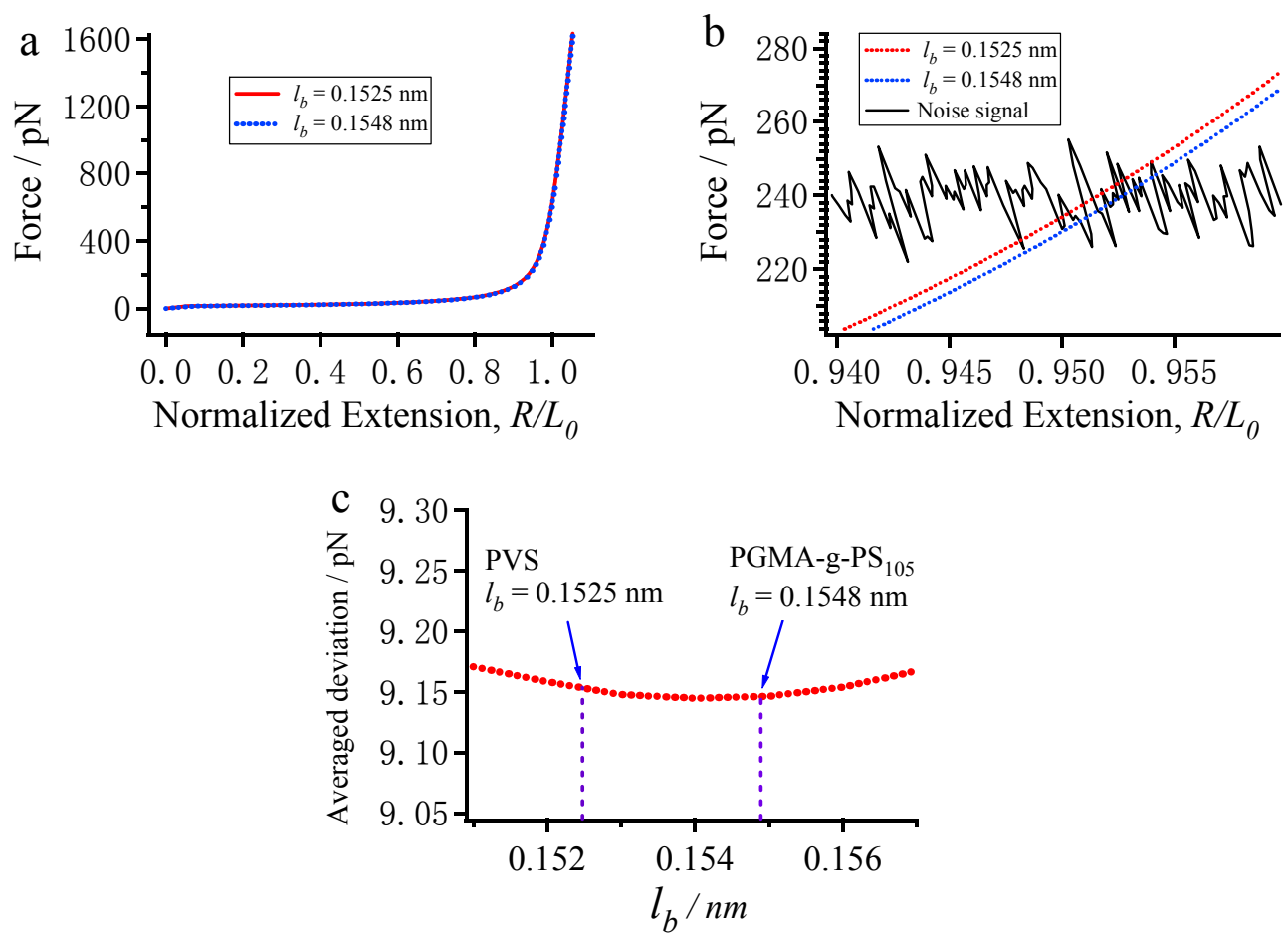

Figure S9. (a) Comparison of QM-FRC fitting curves with $l_{b}=0.1525$ and $0.1548 \mathrm{~nm}$.

(b) The QM-FRC fitting curves are magnified to show the details. For comparison, a typical blank experimental F-E curve is also plotted to show the noise level of SMFS. The differences between the two QM-FRC fitting curves with $l_{b}=0.1525 \mathrm{~nm}$ and $0.1548 \mathrm{~nm}(\sim 3 \mathrm{pN})$ are much smaller than the noise level of SMFS $(\sim 10 \mathrm{pN})$. The small difference of $l_{b}$ from one sample to another (in the range of $0.1525-0.1548 \mathrm{~nm}$ ) should be mainly caused by the thermal fluctuation of the system. (c) Magnified curve of Figure S6d. The small variation of $l_{b}$ around the center $(0.154 \mathrm{~nm})$ makes almost no difference for the fitting performance. 


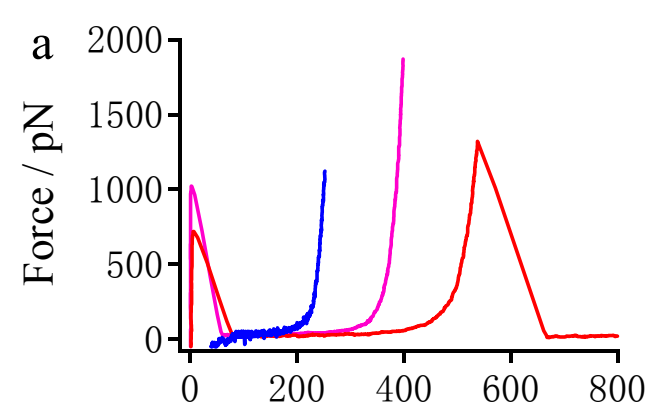

Normalized Extension, $R / L_{0}$

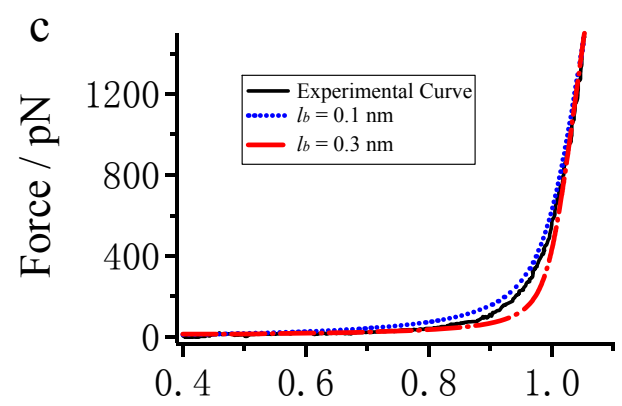

Normalized Extension, $R / L_{0}$

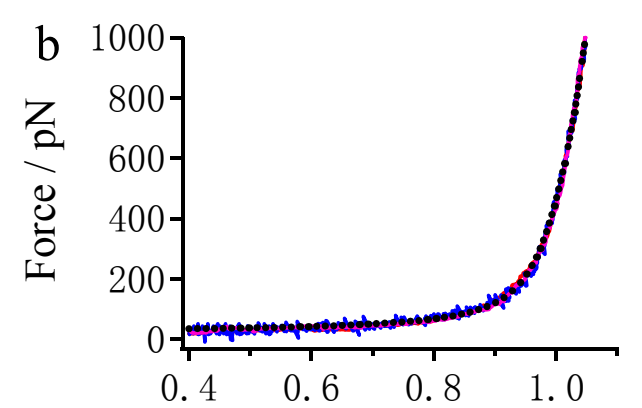

Normalized Extension, $R / L_{0}$

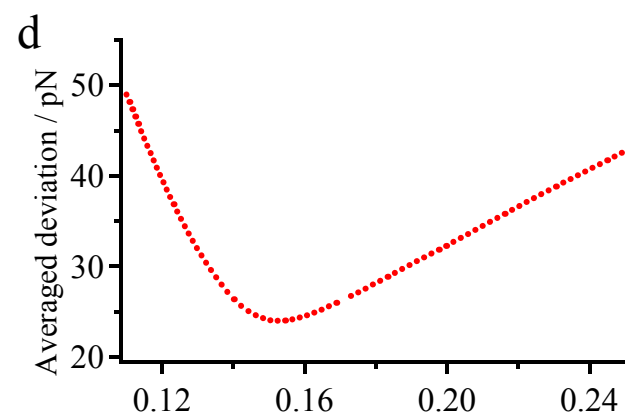

Normalized Extension, $R / L_{0}$

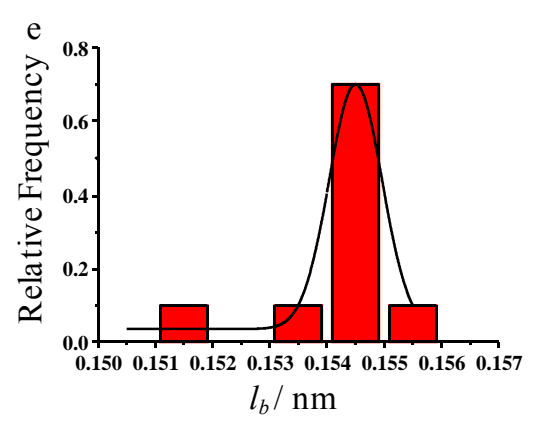

Figure S10. (a) The F-E curves of PG1 obtained in nonane. (b) The normalized F-E curves of those shown in (a) and the QM-FRC fitting curve of PE $\left(l_{b}=0.1545 \mathrm{~nm}\right.$, dotted line). (c) Normalized single-molecule force curve of PG1 vs QM-FRC fitting curves with various $l_{b}$ values. (d) Averaged deviation of force between the experimental F-E curve of PG1 showing in (c) and the QM-FRC fitting curve in the range of $R / L_{0}=0.5-1.05$. The minimum deviation is obtained at $l_{b}=0.153 \mathrm{~nm}$. (e) The histogram of $l_{b}$ of all F-E curves of PG1 obtained in nonane. Gaussian fit shows that the most probable of $l_{b}$ is $0.1545 \mathrm{~nm}$ with a standard error of $1.3 \times 10^{-4} \mathrm{~nm}$. 


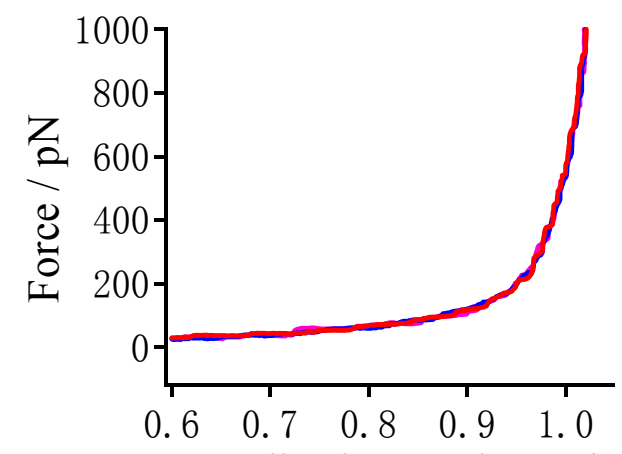

Normalized Extension, $R / L_{0}$

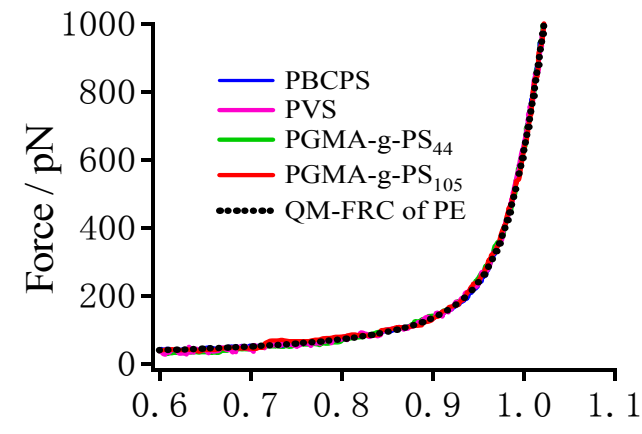

Normalized Extension, $R / L_{0}$

Figure S11. To display the details of the F-E curves, Figures 3b (left) and 5a (right) are magnified here.

\section{The details of the QM-FRC model fitting.}

In the $\mathrm{FRC}$ model, ${ }^{2,3}$ the relationship between the stretching force $(F)$ and the extension $(R)$ of the chain can be written as

$$
R=L[F]\left[1-k_{B} T /\left(2 F l_{b}\right)\right]
$$

In Eq S.2, $k_{B}$ is the Boltzmann constant, $T$ is temperature, $l_{b}$ is the length of the rotating unit, $F$ is the stretching force acted on the polymer chain, $L[F]$ is the contour length of the chain at a given $F$, and $R$ represents the extension of the polymer chain (end-to-end distance).

Note that $L[F]$ increases with the increasing of force. Considering that the bond angles and bond lengths are increased upon elongation, the quantum mechanical (QM) calculations are used to obtain the theoretical elasticity of the polymers with a C-C backbone. ${ }^{5}$ In the QM calculations, the polymer chain only has $\mathrm{H}$ atoms as 'side chains'. These results have been used successfully to describe the single-molecule 
elasticity of the polymers with C-C backbones (PDEAm, PAAm and PS). ${ }^{5}$ This result suggests that the influence of the side chains on the polymer SCEE of these polymers can be ignored. Therefore, for most polymers with a $\mathrm{C}-\mathrm{C}$ backbone, we hypothesize that the SCEE will not be influenced by the side chains. Thus, we can use the same elastic modulus below:

$$
\begin{aligned}
& F=\sum_{n=1}^{3} \gamma_{n}\left(L[F] / L_{0}-1\right)^{n} \\
& \quad \gamma_{1}=28.7 \mathrm{nN}, \gamma_{2}=-42.0 \mathrm{nN}, \gamma_{3}=16.9 \mathrm{nN}
\end{aligned}
$$

where $L_{0}$ is the contour length of the polymer chain at zero force. Therefore, if we normalize the end-to-end distance by $L_{0}$, the model can be modified as

$$
R / L_{0}=\left(L[F] / L_{0}\right) \cdot\left[1-k_{B} T /\left(2 F l_{b}\right)\right]
$$

where $R / L_{0}$ is the normalized extension of a polymer chain. The details of this model can be found in the reference. ${ }^{2-5}$ In brief, $L[F] / L_{0}$ increases with increasing $F$ ( $F$ can be calculated by Eq S.3) until the breakage of the polymer bridge. Thus, the model has only one parameter $\left(l_{b}\right)$ left. The modified FRC model (Eq S.4), which is integrated with the QM results, is called the QM-FRC model. Taking into account that the molecular structure of PG2 has the same $\mathrm{C}-\mathrm{C}$ backbone with the previously studied polymers, ${ }^{3}$ we also use the result $\left(l_{b}=0.154 \mathrm{~nm}\right)$ to obtain the fitting curve, because the $\mathrm{C}-\mathrm{C}$ bond length is represented by the value of $l_{b}$. Thus, a QM-FRC fitting curve can be generated, see Figure 4. However, it is surprising that there is a marked deviation between the fitting curve and the F-E curve of PG2. This result implies that the SCEE of PG2 is strongly influenced by its G2 side chains. 
In the QM-FRC model, the elastic moduli are obtained from PE. However, the size of G2 side chain is much larger than the $\mathrm{H}$ atoms of PE. Thus, we think that the marked difference between the fitting curve and the F-E curve of PG2 should be caused by the QM calculations. Note that there are three coefficients in the QM elastic modulus, $\gamma_{1}, \gamma_{2}$ and $\gamma_{3} . \gamma_{1}$ is the linear elastic modulus, $\gamma_{2}$ and $\gamma_{3}$ are nonlinear corrections which become significant in the higher force region. Therefore, it is expected that we can obtain a new set of elastic moduli $\left(\gamma_{1-M}, \gamma_{2-M}\right.$ and $\left.\gamma_{3-M}\right)$ for PG2 through the way of modifying the values of $\gamma_{1}, \gamma_{2}$ and $\gamma_{3}$ on the basis of $l_{b}=0.154$ $\mathrm{nm}$ (due to the C-C backbone of PG2). To obtain an optimum fitting curve, we adjust the $\gamma$ values over and over again. Finally, one of the optimal solutions was obtained, see Table S2. With the modified elastic moduli $\left(\gamma_{1-M}, \gamma_{2-M}\right.$ and $\left.\gamma_{3-M}\right)$, a new fitting curve, named QM-FRC $\mathrm{Q}_{M}$ fitting curve, has been obtained (Figure $\mathrm{S} 12 \mathrm{a}$ ). It is expected that there are several sets of $\gamma$ values that can generate acceptable fitting results. We only provide one of them in the manuscript.

Table S2. The elastic moduli $\left(\gamma_{1}, \gamma_{2}\right.$ and $\left.\gamma_{3}\right)$ obtained from the QM calculations ${ }^{3}$ in the QM-FRC model and the modified elastic moduli $\left(\gamma_{1-M}, \gamma_{2-M}\right.$ and $\left.\gamma_{3-M}\right)$ in the QM-FRC $\mathrm{Q}_{M}$ model.

\begin{tabular}{|c|c|c|c|}
\hline \multicolumn{2}{|c|}{ QM-FRC model } & \multicolumn{2}{c|}{ QM-FRC ${ }_{M}$ model } \\
\hline$\gamma_{1}$ & $28.7 \mathrm{nN}$ & $\gamma_{1-M}$ & $45.9 \mathrm{nN}$ \\
\hline$\gamma_{2}$ & $-42.0 \mathrm{nN}$ & $\gamma_{2-M}$ & $-336.0 \mathrm{nN}$ \\
\hline$\gamma_{3}$ & $16.9 \mathrm{nN}$ & $\gamma_{3-M}$ & $3380.0 \mathrm{nN}$ \\
\hline
\end{tabular}



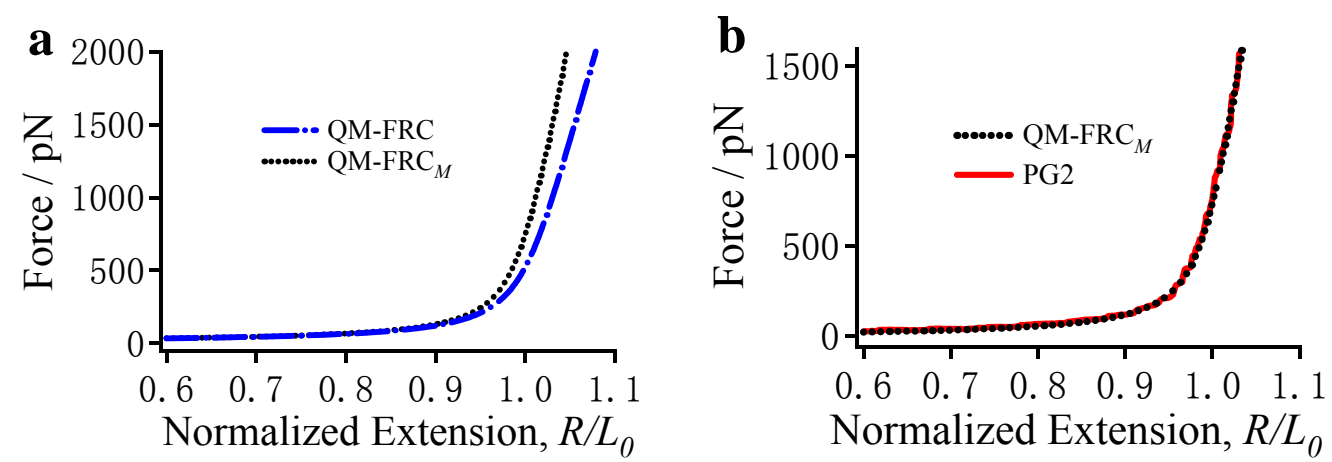

Figure S12. Comparison of two kinds of fitting curves. (a) The two different fitting curves. The blue dotted line is the QM-FRC fitting curve $\left(\gamma_{1}=28.7 \mathrm{nN}, \gamma_{2}=-42.0 \mathrm{nN}\right.$ and $\left.\gamma_{3}=16.9 \mathrm{nN}\right)$ and the black dotted line is the QM-FRC ${ }_{M}$ fitting curve $\left(\gamma_{1-M}=45.9\right.$ $\mathrm{nN}, \gamma_{2-M}=-336.0 \mathrm{nN}$ and $\gamma_{3-M}=3380.0 \mathrm{nN}$ ). (b) The normalized F-E curve of G2 (red) and the QM-FRC $\mathrm{C}_{M}$ fitting curve (dotted line).

From Figure S12b, we can see that the F-E curve of PG2 can be described well by the QM-FRC model with the modified elastic moduli. This good fitting result indicates the modified elastic moduli may present the inherent elasticity of PG2. This artificial fitting method is practical since accurate QM calculations are difficult for PG2 at present.

\section{References}

1. Wang, C.; Shi, W.; Zhang, W.; Zhang, X.; Katsumoto, Y.; Ozaki, Y. Force Spectroscopy Study on Poly(acrylamide) Derivatives: Effects of Substitutes and Buffers on Single-Chain Elasticity. Nano Lett. 2002, 2, 1169-1172. 
2. Livadaru, L.; Netz, R. R.; Kreuzer, H. J. Stretching Response of Discrete Semiflexible Polymers. Macromolecules 2003, 36, 3732-3744.

3. Cui, S. X.; Yu, Y.; Lin, Z. B. Modeling Single Chain Elasticity of Single-Stranded DNA: A Comparison of Three Models. Polymer 2009, 50, 930-935.

4. Hugel, T.; Rief, M.; Seitz, M.; Gaub, H. E.; Netz, R. R. Highly Stretched Single Polymers: Atomic-Force-Microscope Experiments Versus ab-initio Theory. Phys. Rev. Lett. 2005, 94, 048301.

5. Wang, K. F.; Pang, X. C.; Cui, S. X. Inherent Stretching Elasticity of a Single Polymer Chain with a Carbon-Carbon Backbone. Langmuir 2013, 29, 4315-4319. 